\title{
A tactile illusion: The rotating hourglass
}

\author{
KAREN N. JONES* \\ University of Oklahoma, Norman, Oklahoma 73069 \\ R. MARK TOUCHSTONE \\ Civil Aeromedical Institute, FAA, Oklahoma City, Oklahoma 73125 \\ and \\ CHARLES F. GETTYS \\ University of Oklahoma, Norman, Oklahoma 73069
}

\begin{abstract}
A new tactile (more properly termed haptic) illusion, the rotating hourglass, was investigated in the laboratory by rotating a rod end for end between the S's thumb and forefinger. This illusion, which is an apparent decrease in the diameter of the rod at the point of contact with the fingers, was easily observed by 19 of the 20 Ss. When the illusion was studied as a function of time, the magnitude of the illusion increased over time with a mean decrease in apparent diameter of $52.3 \%$ from the beginning to the end of the 38-sec trials. A theory of differential adaptation of the skin is postulated to explain the rotating hourglass illusion and a similar illusion.
\end{abstract}

This illusion, which we call the rotating hourglass, is an apparent decrease in the diameter of the part of a rotating rod which is in contact with the finger. Most people can experience a weak version of this illusion by using a piece of chalk. To obtain the illusion, hold the chalk in the center with the thumb and forefinger. Then steadily rotate the piece of chalk end over end with the other hand so that each end passes through the circle formed by the thumb and forefinger. While rotating the chalk end for end at $30-50 \mathrm{rpm}$, grip it fairly firmly, keep your fingers in the same location on the piece of chalk, and concentrate on the tactile sensations produced by the piece of chalk. After a short period of time, the diameter of the chalk should appear to decrease where it is in contact with the holding fingers.

The purpose of this experiment was to investigate the rotating hourglass illusion in the laboratory under more controlled conditions. A tentative theory suggested that adaptation to the sensation of pressure produced by tissue movement was a probable explanation for the illusion. The rate of tissue movement over time is a negatively accelerated function of depth of deformation (Nafe \& Wagoner, 1941a). The authors felt that, if the illusion was to be explained by a theory of pressure adaptation of the skin, the study of the time-course of the illusion was important and the rate of rotation of the rod might have an effect on the extent of the illusion.

\section{EXPERIMENT I}

\section{Method \\ Apparatus. To gain better stimulus control, an apparatus was constructed to rotate a rod between S's thumb and forefinger. A}

* Requests for reprints should be sent to Karen $N$. Iones, Department of Psychology, University of Oklahoma, Norman, Oklahoma 73069 . schematic drawing of this apparatus is shown in Fig. 1. A steel rod (a), $6.35 \mathrm{~mm}$ in diam, was mounted in a flat ring (b), $1.86 \mathrm{~cm}$ wide with an outside diameter of $5.67 \mathrm{~cm}$ and an inside diameter of $5.60 \mathrm{~cm}$. The ring was mounted in three flanged rollers, which were tangent to the ring at three places around its circumference. Two of the rollers (c) served as idlers; the third roller (d) was driven by a variable-speed motor (e), which could turn the ring containing the rod at a calibrated rpm. S's thumb and forefinger encircled, but did not touch, the ring holding the rod when the thumb and forefinger were in contact with the rod.

A schematic drawing of the tracking apparatus used to make measurements of the apparent diameter of the rod over time is shown in Fig. 2. Two metal rods (a), each with a small metal tube (b) attached at one end, were connected at a pivot point. Ss adjusted the distance between the two tubes with the thumb and forefinger until this distance was subjectively equal to the

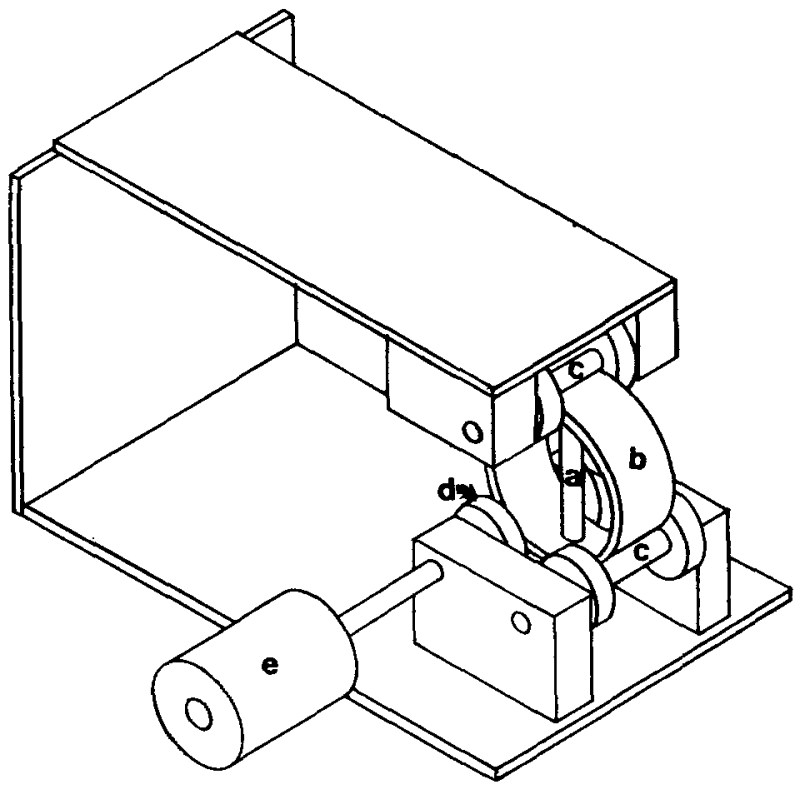

Fig. 1. Schematic drawing of the apparatus used for presentation of stimuli. 


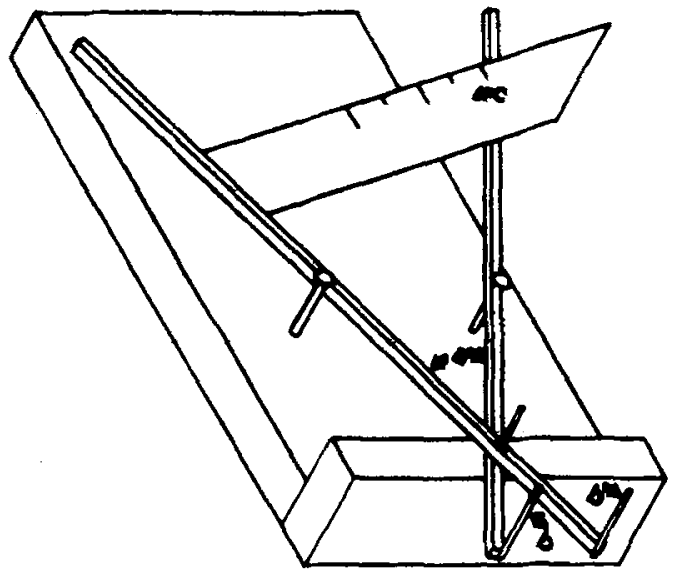

Fig. 2. Schematic drawing of the tracking apparatus used for measuring the apparent diameter of the stimulus as a function of time.

illusory diameter of the rotating rod at its smallest point. Movement of the metal tubes produced the movement of one of the rods along a scale (c) at tached to the other rod. The scale was calibrated to allow recording of the distance between the outside walls of the tubes and hence S's estimate of the apparent diameter of the rotating rod.

All of the apparatus was concealed from S's view by a screen. $S$ was seated in front of the screen so that he could comfortably insert his right hand through a curtained window to grasp the rotating rod and also insert his left hand through a curtained window to use the tracking device. $\mathrm{E}$ was seated behind the screen directly across from $S$. The screen allowed $E$ to make recordings without disturbing $S$.

Subjects. Twenty right-handed students in an introductory psychology class served as $S$ s in this experiment as partial fulfillment of a course requirement.

Design and Procedure. The data were collected in two phases. The purpose of the first phase was to demonstrate that these Ss, without prompting as to the nature of the illusion, experienced the illusion. S was told that he would hold a rod-shaped rotating object which might appear to change in size or shape by becoming either larger or smailer. Then $\mathrm{S}$ held the rolating $(40 \mathrm{rpm})$ rod between the thumb and forefinger of his right hand. After reporting any apparent change to $E, S$ was asked to sketch on a card where and by what relative amount the rod appeared to change. Two parallel lines representing the walls of the cylinder were drawn on the card $1.3 \mathrm{~cm}$ apart.

In the second phase of the experiment, changes in the illusion were studied as a function of time. The tracking apparatus shown in Fig. 2 was used because it allowed continuous recording of S's estimate of the size of the rod and allowed S to make his estimations of the illusion within the same sense modality. First, using the tracking device, $S$ made a series of size estimations of the diameter of six motionless metal rods ranging in size from 3.2 to $7.11 \mathrm{~mm}$. The purpose of this was to establish a relationship between known physical stimuli and the S's size estimation. The calibration procedure demonstrated that S's size estimation was a linear function of the size of the six comparison stimuli. At the start of each trial, $S$ equated the distance between the metal tubes of the tracking device with the diameter of the nonrotating rod to give a baseline measure. Then the rod was put in rotation and $S$ constantly equated the apparent distance between the tubes with the apparent diameler of the rotating rod at its smallest point. Four trials at each of three speeds of rotation $(30,40$, and $50 \mathrm{rpm})$ were presented to $S$ in random order. Each trial had a duration of $38 \mathrm{sec}$ and measurements of the apparent diameter of the stimulus were recorded just before the start of each trial, $5 \mathrm{sec}$ after the beginning of rotation of the rod and then every 10 sec until the end of the trial.

\section{Results and Discussion}

During the first phase, 19 of the 20 Ss spontaneously reported that the rod seemed to become smaller where their fingers were touching it. The drawings made by the first 4 of the Ss, which are representative of drawings made by the 19 Ss experiencing the illusion during this phase, are shown in Fig. 3. The average decrease in apparent diameter for all 20 drawings was $52.3 \%$.

The size of the mean decrease in the apparent diameter of the rod for each rpm at various times is shown in Fig. 4. A three-way analysis of variance of this second phase data with all factors as repeated measures indicated a significant decrease in the apparent diameter of the rod as a function of time $(F=65.7$; $\mathrm{df}=3,57$; $\mathrm{p}<.0001)$. The mean decrease in the apparent diameter of the rod for all rpms from the beginning to the end of the trial was $.21 \mathrm{~cm}$. The effect of rpm was small and nonsignificant $(\mathrm{F}<1.0){ }^{1}$

We hypothesize that the subjective decrease in diameter experienced by Ss is the result of the following factors. The sensation of pressure apparently is generated by the deformation of tissue. When the rate of tissue movement drops below a threshold value, the sensation of pressure disappears (Kenshalo, 1971). Since at the exact center of rotation of the rod the skin is in relatively constant contact with the rod and the movement of tissue is comparatively small or

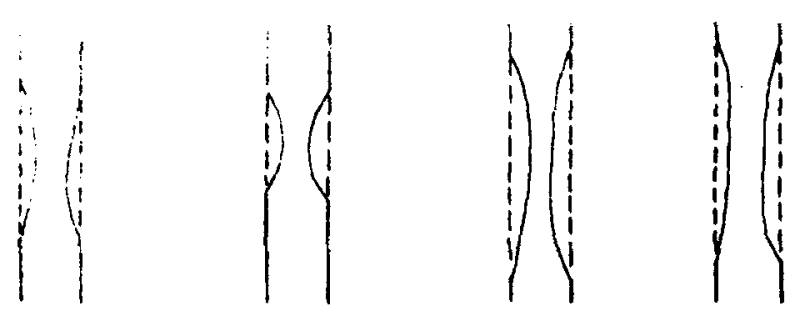

Fig. 3. Drawings made by the first four Ss after experiencing the rotating hourglass illusion.

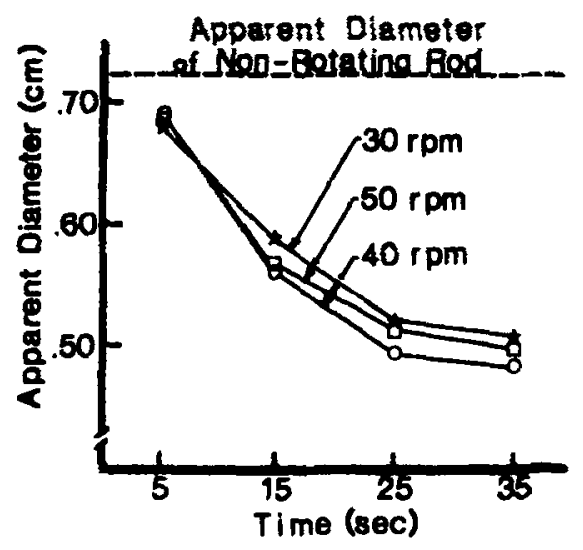

Fig. 4. Mean change in apparent diameter of rod at 30, 40, and $50 \mathrm{rpm}$ as a function of time for all $20 \mathrm{Ss}$. 
nonexistent, partial or complete adaptation to the sensation of pressure occurs. Points on the skin some distance away from this center receive intermittent stimulation as the rod sweeps across them; consequently, tissue deformation never reaches asymptote and the sensation of pressure persists and is greater at peripheral points than at the center. Also, the deformation of the skin on the finger produced by the moving rod becomes more transitory as distance from the center of rotation increases. Given the rather sluggish elastic and plastic properties of the skin, the more transitory the stimulation, the more time the skin has to recover from each deformation before the next pass of the rod, resulting in a greater tissue movement at these distant points. This effect should enhance the apparent pressure differential produced by the moving rod. We hypothesize that a motionless hourglass-shaped rod produces a highly similar pressure pattern, except of course that it is not in motion. At the center of the stationary hourglass, the sensation of pressure is low, and the pressure steadily increases as the distance between a given point on the skin and the center of the hourglass increases. In the rotating hourglass illusion, therefore, the moving rod produces about the same pattern of pressure as that produced by a stationary hourglass, and the pattern of stimulation is perceived as such.

The decrease in apparent diameter of the rod as a function of time is in agreement with this adaptation theory. Since the skin does not adapt to constant stimulation immediately, the magnitude of the illusion increases as the skin at the center of rotation adapts to the continuous stimulation and the amount of perceived pressure at the center of rotation decreases in comparison with the amount of perceived pressure at other points on the two fingers. The deformation of the skin to pressure occurs rapidly at first and then more slowly until the asymptotic value is reached (Kenshalo, 1971). Consequently, the illusion would be expected to increase rapidly at first and then more slowly with time. The data shown in Fig. 4 support this prediction. Nafe and Wagoner (1941b) report data showing that adaptation occurs after approximately $5-25 \mathrm{sec}$, depending on the amount of pressure. The data presented in Fig. 4 suggest that the hourglass illusion approaches asymptote at approximately $25-35 \mathrm{sec}$. Given the fact that the rod was moving, the time-course of the illusion is in reasonable agreement with Nafe and Wagoner's data.

This theory can explain the hourglass illusion and perhaps qualitatively predict the perception produced by other forms of stimulation. However, at present, quantitative predictions are difficult to make because not enough is known about the time course of adaptation of the haptic sense under static and dynamic stimulation.

\section{EXPERIMENT II: A SIMILAR ILLUSION}

While this paper was in preparation, Cormack (1973) reported a similar illusion. Cormack's illusion is obtained by turning a disk edge for edge between the thumb and forefinger. The diameter of the disk appears to elongate for the turning hand. Cormack hypothesized that the illusion was caused by adaptation "... to the continual pressure and twisting on the finger tips [p. 591]." Since the fingers of the turning hand receive a different pattern of stimulation, one which does not lead to adaptation, Cormack hypothesized that his illusion arose as a result of differential adaptation between the two hands. He also proposed an alternative theory that the effect arises from a differential adaptation to range of separation between the fingers of the two hands. The holding fingers are always separated by the diameter of the coin, but the turning fingers change their separation from the diameter to the thickness of the coin in the process of turning the coin. "Thus, the turning fingers became adapted to a narrower average separation than the holding fingers. This differential adaptation may lead to the difference in apparent length for the two hands [pp. 591-592]." While Cormack did not specify any exact adaptation mechanisms operating in either of his two theories, both theories hypothesized that the effect was due to differential adaptation between the fingers of the two hands. As the rotating hourglass illusion can be explained by differential adaptation within one finger of the holding hand only, we decided it would be interesting to attempt to replicate Cormack's result, confining stimulation to only one hand.

\section{Method}

Four Ss were used to demonstrate that Cormack's illusion could be replicated using the same method and apparatus we had used to demonstrate and measure the rotating hourglass illusion. $S$ held a revolving disk (a quarter rotating at $50 \mathrm{rpm}$ ) between the thumb and forefinger of his right hand until the stimulus appeared to change. The $S$ was asked to sketch where and how the disk had appeared to change on a card on which a circle was drawn which was the same size as the disk used. Then the tracking apparatus was used to make measurements of the change in the apparent diameter of the disk as a function of time.

\section{Results and Discussion}

Ss' drawings (reproduced in Fig. 5) indicated that the diameter of the disk between the fingers holding the coin was exceeded by an average of $56.4 \%$ by the diameter of the other dimension. All of Ss' drawings show the paradoxical result that the dimension of the disk they were not holding appeared to elongate. Two Ss' drawings indicated that the diameter of the disk between the fingers appeared to decrease. Our technique of instructing $S$ to sketch the maximum extent of his illusion is roughly comparable to Cormack's measurement technique. Cormack's method of 

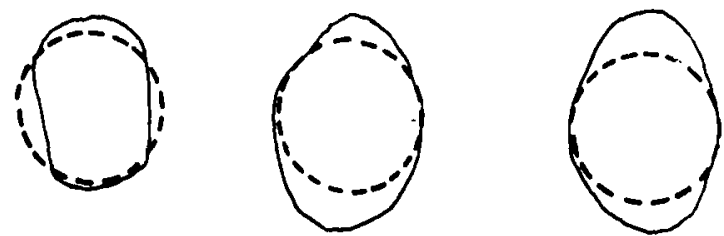

measurement consisted of presenting $S$ with a series of oblong drawings that varied in their length-to-width ratio and instructing $S$ to continually pick the drawing that most closely corresponded to his haptic experience at that time. Our Ss' drawings showed a mean decrease of $52.3 \%$ for the hourglass illusion (at $40 \mathrm{rpm}$ ) and $56.4 \%$ for Cormack's illusion (at $50 \mathrm{rpm}$ ). Cormack reports a mean decrease of $62 \%$ under similar circumstances $(50 \mathrm{rpm})$ for his Ss.

When we measured Cormack's illusion using our apparatus, there was a mean decrease of $.17 \mathrm{~cm}$ from the beginning to the end of the $38-\mathrm{sec}$ trials. The rotating hourglass illusion yielded a mean decrease of $.21 \mathrm{~cm}$. Given the difference between the stimuli used to produce the illusion in each case, these two measures are in good agreement. The similarity of the results suggests that both illusions are caused by the mechanism of differential adaptation within each of the fingers of one hand.

The results of our study indicate that the data obtained are very dependent on the measurement technique used. When $S$ is asked to make a visual representation of the tactile illusion, the measured magnitude of the illusion is greater than when $S$ makes a haptic judgment of the illusion. Using a given measurement technique, good agreement is obtained between the magnitude of the two illusions. This agreement indicates that both the rotating hourglass illusion and the illusion demonstrated by Cormack have

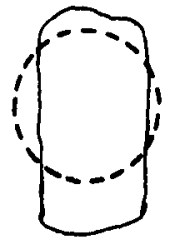

Fig. 5. Drawings made by the four Ss after experiencing the illusion demonstrated by Cormack. Ss were asked to assume that the quarter was rotating with the axis of rotation being horizontal. the same underlying mechanism, which we hypothesize as being differential adaptation of the skin.

\section{REFERENCES}

Cormack, R. H. Haptic illusion: Apparent elongation of a disk rotated between the fingers. Science, 1973, 179, 590-592.

Kenshalo, D. R. The cutaneous senses. In J. W. Kling and L. A. Riggs (Eds.), Woodworth and Schlosberg's Experimental psychology. New York: Holt, Rinehart \& Winston, 1971. Pp. 117-168.

Nafe, J. P., \& Wagoner, K. S. The nature of sensory adaptation. Journal of General Psy chology, 1941a, 25. 295-321.

Nafe, J. P., \& Wagoner, K. S. The nature of pressure adaptation. Journal of General Psychology, 1941b, 25, 323-351.

\section{NOTE}

1. Several other variables were qualitatively investigated with four Ss. With the rod rotating at $40 \mathrm{rpm}$, each $S$ was asked to (1) move his fingers slightly vertically and then horizontally: (2) while experiencing the illusion. look at the rod (previously all of the apparatus had been shielded from S's view); and (3) hold only his forefinger on the rotating rod. The results indicated that: (1) the illusion disappears at least momentarily when the fingers are moved: (2) when the illusion is experienced, the rod does not appear to change visually; (3) the side of the rod appears to indent like one side of an hourglass when only one finger is held against the rotating rod. An additional four Ss were also tested to determine if the illusion could be experienced with a stationary rod. $S$ was asked to estimate the size of a rod using the tracking apparatus for four 38-sec trials. Each $\mathbf{S}$ showed slight random variations in his estimations over time, but none mentioned experiencing any change in the size or shape of the rod.

(Received for publication August 27, 1973; revision received November $23,1973$. ) 